\title{
Silica glass-based fiberoptic distributed meandering waveguides for integrated photonics
}

Muhammad Zakwan, Ceren B. Dağ, Ali Serpengüzel

Muhammad Zakwan, Ceren B. Dağ, Ali Serpengüzel, "Silica glass-based fiberoptic distributed meandering waveguides for integrated photonics," Proc. SPIE 10683, Fiber Lasers and Glass Photonics: Materials through Applications, 1068309 (17 May 2018); doi: 10.1117/12.2314966 


\title{
Silica glass-based fiber optic distributed meandering waveguides for integrated photonics
}

\author{
Muhammad Zakwan, ${ }^{\text {a,b* }}$ Ceren B. Dağ, ${ }^{c}$ and Ali Serpengüzel ${ }^{\mathrm{a}}$ \\ ${ }^{a}$ Koç University, Department of Physics, Microphotonics Research Laboratory, Rumelifeneri Yolu, \\ Sarıyer, Istanbul 34450 Turkey \\ ${ }^{\mathrm{b}}$ Air University, Department of Avionics Engineering, \\ Sector E-9, Islamabad 44000 Pakistan \\ ${ }^{\mathrm{c}}$ University of Michigan, Department of Physics, 1440 Randall Laboratory, 450 Church Street, \\ Ann Arbor, Michigan 48109 USA
}

\begin{abstract}
Distributed meandering waveguide based fiber optic components are introduced, categorized, and numerically analyzed in the near-infrared. The building block of all meandering waveguide components is the meandering loop mirror. The other components are the meandering resonator, meandering distributed feedback structure, symmetric and antisymmetric meandering resonator, symmetric and antisymmetric meandering distributed feedback structures giving rise to transmission spectra with Lorentzian, Rabi, Fano, coupled resonator induced transparency, and winged Lorentzian lineshapes. With this variety of spectral responses, distributed meandering waveguide fiber optic components are suitable as filters, and delay line elements in fiber optic communication, and as sensor elements in fiber optic diagnostics.
\end{abstract}

Keywords: fiber optics systems, optical interconnects, tunable filters, sensors, waveguide devices.

\section{INTRODUCTION}

Resonators have proven to be a key element in the basic building blocks of optical components. In fiber optic based systems resonators are widely used for realizing sensors [1] and manufacturing allfiber lasers [2]. Over the past years optical resonators have also gained popularity in the SOI based systems in the form of ring resonator [3] and microdisk resonator [4]. Meandering structures can be used as resonators for wide range of microwave circuits elements such as filters [5], delay lines [6], and antennas [7]. Meandering structures have been used in single [8,9], and analyzed in double [10], and multiple [11] loop mirrors in fiber optics [12]. Recently, single [13] and double [14] selfcoupled optical waveguides (SCOWs) have been realized. Coupled meandering waveguides [15] have been analyzed in planar lightwave circuits. In our recent studies, we introduced, categorized, and numerically analyzed the intensity [16], phase [17], and phasor [18] response of distributed meandering waveguides (DMWs) applied to of silicon-on-insulator (SOI) components. Here we are introducing, categorizing, and numerically analyzing DMWs applied to single mode fiber (SMF) components, for the first time to the best of our knowledge, for use in fiber optic communication and diagnostics [19]. 

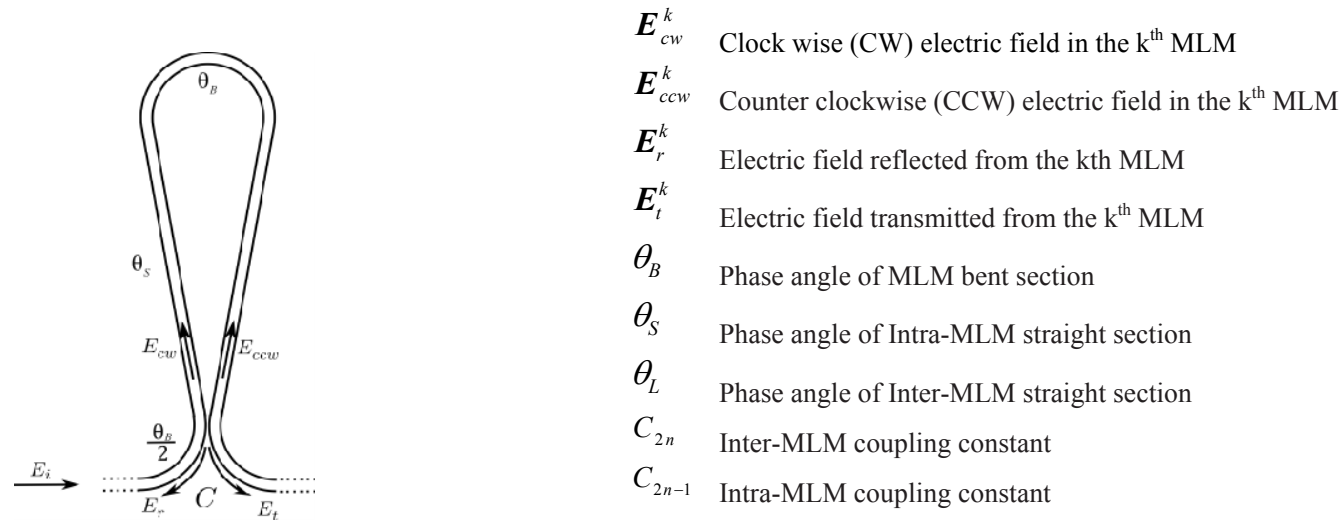

Fig. 1. MLM indicating bent section phase $\theta_{B}$, coupling constant $C$, straight section phase $\theta_{S}$, and the definitions of DMW parameters.

\section{MEANDERING LOOP MIRROR}

The meandering loop mirror (MLM) [20] is a waveguide bent so that, it is coupled back to itself with an intra-MLM coupling constant $\mathrm{C}$ as shown in Fig. 1. MLM has widely been used in Sagnac interferometers for optical gyroscope [21] and delay line [22] applications. In our analysis, the bent section comprise of a semicircle, such that, the wave, after propagating through the bent section, acquires an optical phase of $\theta_{B}$. Similarly the wave, after passing through one of the two intra-MLM straight sections, accumulates a phase $\theta_{S}$ as shown in Fig. 1. During our calculations, we assume $\theta_{B}$ to be equal to $\theta_{S}$ for the sake of simplicity. Using the coupled field analysis, we can express $\mathrm{E}_{\mathrm{cw}}$ and $\mathrm{E}_{\mathrm{ccw}}$ into the reflected and transmitted electric fields [16].

The typical SMF has an effective refractive index of around $\mathrm{N}_{\text {eff }}=1.468$, with typical core and cladding diameters of $9 \mu \mathrm{m}$ and $125 \mu \mathrm{m}$, respectively [23]. A SMF with bend radii of $5 \mathrm{~mm}, 7.5 \mathrm{~mm}$, and $10 \mathrm{~mm}$ can suffer from bending losses of $0.15 \mathrm{~dB} /$ turn, $0.08 \mathrm{~dB} /$ turn, and $0.03 \mathrm{~dB} / \mathrm{turn}$, respectively [24]. The corresponding straight section lengths of the MLM turn out to be $5 \pi \mathrm{mm}, 7.5 \pi \mathrm{mm}$, and $10 \pi \mathrm{mm}$, respectively. As shown in Fig. 1 an MLM has a couple of straight and bent sections, corresponding to a total parameter of the MLM of $20 \pi \mathrm{mm}, 30 \pi \mathrm{mm}$, and $40 \pi \mathrm{mm}$ resulting in footprints of $12 \times 20 \mathrm{~mm}^{2}, 18 \times 30 \mathrm{~mm}^{2}$, and $24 \times 40 \mathrm{~mm}^{2}$ for bend radii of $5 \mathrm{~mm}, 7.5 \mathrm{~mm}$, and $10 \mathrm{~mm}$, respectively.

These footprints can be further reduced by optimizing the length of the straight section and the bend radius of the MLM. In addition, typical coupling losses of SMFs are on the order of $0.15 \mathrm{~dB}$ [25]. The standard attenuation losses of the SMFs due to the straight section are negligible $(\leq 0.35 \mathrm{~dB} / \mathrm{km})$. The bending losses of $0.15 \mathrm{~dB}, 0.08 \mathrm{~dB}$, and $0.03 \mathrm{~dB}$, when added with coupling section's loss aggregate to the total losses of $0.30 \mathrm{~dB}, 0.23 \mathrm{~dB}$, and $0.18 \mathrm{~dB}$ for bend radii of $5 \mathrm{~mm}, 7.5 \mathrm{~mm}$, and $10 \mathrm{~mm}$, respectively, hence putting a bound to the coupling-constant $C$ of the MLM.

\section{MEANDERING RESONATOR}

The meandering resonator (MR), analogous to a Fabry-Perot resonator or a ring resonator, has been realized using MLM's [10] for communication applications. MR is obtained by cascading two MLM sections as shown in Fig. 2, where $\theta_{L}$ is the total phase shift gained, while the wave travels an inter-MLM distance, and $\left(\mathrm{C}_{1} \mid \mathrm{C}_{3}\right)$ are the coupling constants of the two cascaded MLMs. We assume that, the inter-MLM straight section phase $\theta_{S}$ is equal to the intra-MLM straight section phase $\theta_{L}$ for 
the sake of simplicity. For the chosen bend radii of $5 \mathrm{~mm}, 7.5 \mathrm{~mm}$, and $10 \mathrm{~mm}$, the geometric path lengths of the MR turn out to be $50 \pi \mathrm{mm}, 75 \pi \mathrm{mm}$, and $100 \pi$, leading to MR free spectral range (FSR) to be $10.4 \mathrm{pm}, 7 \mathrm{pm}$, and $5.2 \mathrm{pm}$, respectively. For the following analysis, we have chosen the minimum bending radius of $5 \mathrm{~mm}$ only, since it has the minimum optical path length leading to the maximum FSR $=10.4 \mathrm{pm}$.

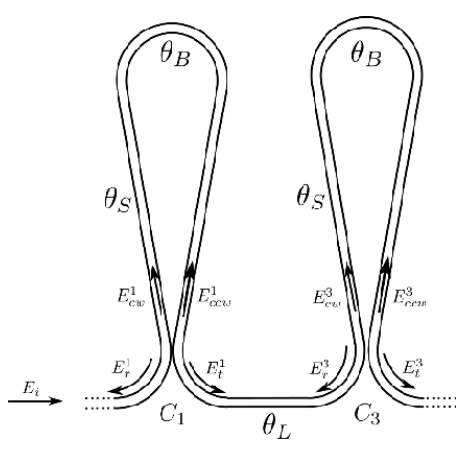

Fig. 2. Two MLM's with $C_{1}$ and $C_{3}$ forming a MR.

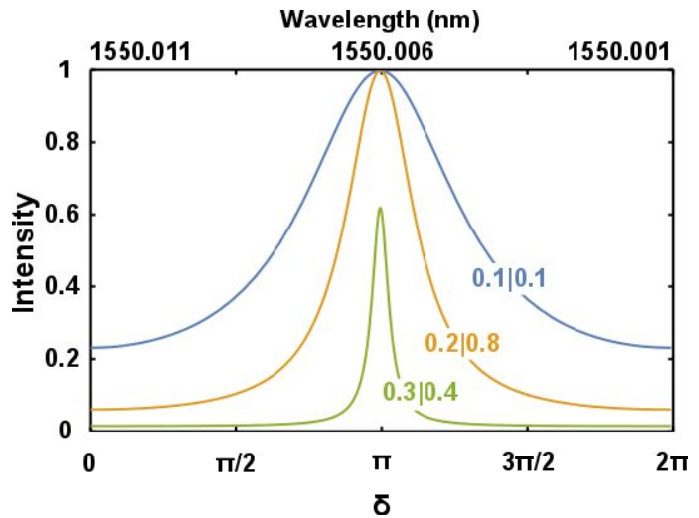

Fig. 3. MR transmittance for $\left(\mathrm{C}_{1} \mid \mathrm{C}_{3}\right)=(0.1 \mid 0.1),(0.2 \mid 0.8)$, and $(0.3 \mid 0.4)$.

For the MR, the coupled electric field analysis [16] results for various values of coupling constants $\left(\mathrm{C}_{1} \mid \mathrm{C}_{3}\right)$ are plotted in Fig. 3, whose spectral coverage is equal to the FSR expressed in terms of accumulated phase angle $(\delta)$ or wavelength $(\lambda)$. We see that, the Lorentzian lineshapes in the transmitted intensity of the MR peaks for the case, when both coupling constants $\left(C_{1} \mid C_{3}\right)$ are equal, i.e., $(0.1 \mid 0.1)$ in Fig. 3. The transmission intensity is symmetric around the coupling constant's value of 0.5 , as we sweep the coupling constant between zero and one. Due to this symmetry, if the coupling constants $\left(\mathrm{C}_{1} \mid \mathrm{C}_{3}\right)$ are set to $(0.2 \mid 0.8)$, we again see the same Lorentzian with a maximum peak value in the transmission intensity, as it would be for the case of coupling constants $(0.2 \mid 0.2)$. For different values of coupling constants $\left(\mathrm{C}_{1} \mid \mathrm{C}_{3}\right)$, the peak intensity drops, when $\left(\mathrm{C}_{1} \mid \mathrm{C}_{3}\right)$ are set to $(0.3 \mid 0.4)$ as shown in Fig. 3.

\section{MEANDERING DISTRIBUTED FEEDBACK STRUCTURE}

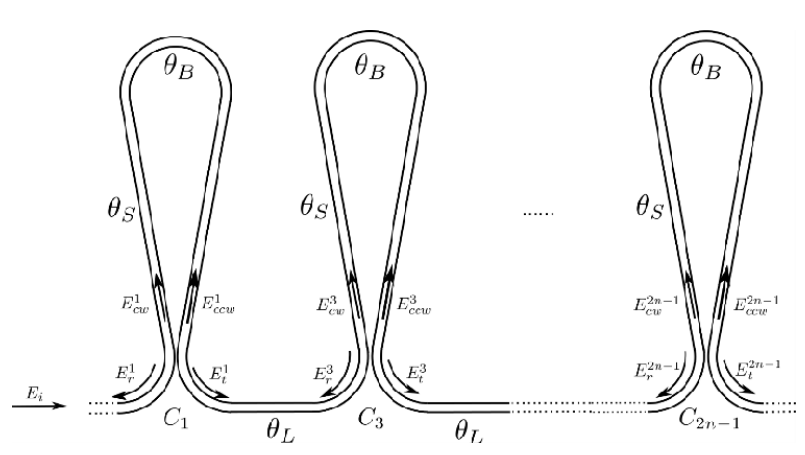

Fig. 4. N cascaded MLM elements forming MDFB structure.

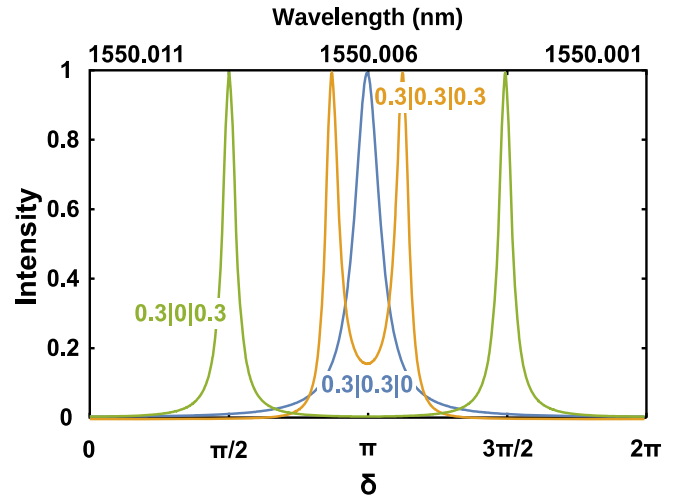

Fig. 5. MDFB of 3 MLM transmittance for $\left(\mathrm{C}_{1}\left|\mathrm{C}_{3}\right| \mathrm{C}_{5}\right)$. 
The meandering distributed feedback structure (MDFB) [11] is an analogue of the 1D photonic crystal (PC) waveguide DBR's proposed using optical fibers [26]. The MDFB in Fig. 4 can be realized by linearly cascading more MLMs to the MR structure. The analysis of MDFB structure can be carried out using the transfer matrix method (TMM) on top of the electric field theory performed for the MR structure.

The FSR for the MDFB is equal to the FSR of the MR with the same MLM dimensions. We chose the number of MLMs to be equal to three for this particular analysis, i.e., $n=3$ leading to the coupling constants $\left(\mathrm{C}_{1}\left|\mathrm{C}_{3}\right| \mathrm{C}_{5}\right)$. In Fig. 5 the transmitted intensities of the MDFB structure are plotted by varying the coupling constants $\left(\mathrm{C}_{1}\left|\mathrm{C}_{3}\right| \mathrm{C}_{5}\right)$. For the case, when $\left(\mathrm{C}_{1}\left|\mathrm{C}_{3}\right| \mathrm{C}_{5}\right)$ are equal to $(0.3|0.3| 0)$, we obtain the same spectrum as in the MR case with $\left(\mathrm{C}_{1} \mid \mathrm{C}_{3}\right)=(0.3 \mid 0.3)$, but as soon as we set the value of $\mathrm{C}_{5}$ in the range $0<\mathrm{C}_{5}<0.5$, we observe mode splitting as is the case for $(0.3|0.3| 0.3)$, similar to the DFB analogy [27] of the FPR, known as mode splitting [28]. Similar to a 1D-PC, the modes of the MDFB circuit create a band stop filter response. Transmission intensity is maximum when $\mathrm{C}_{1}=\mathrm{C}_{5}$, whereas the mode splitting can be controlled by tuning the coupling constant $\mathrm{C}_{3}$ as depicted in Fig. 5, when $\left(\mathrm{C}_{1}\left|\mathrm{C}_{3}\right| \mathrm{C}_{5}\right)$ are changed from $(0.3|0.3| 0.3)$ to $(0.3|0| 0.3)$.

\section{SYMMETRIC MEANDERING RESONATOR}

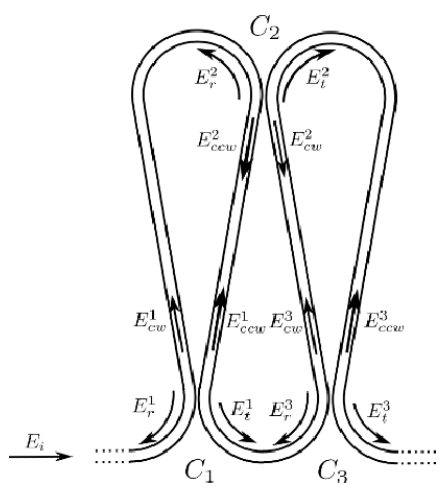

Fig. 6. SMR with $\mathrm{C}_{1}$ and $\mathrm{C}_{3}$, coupled with $\mathrm{C}_{2}$.

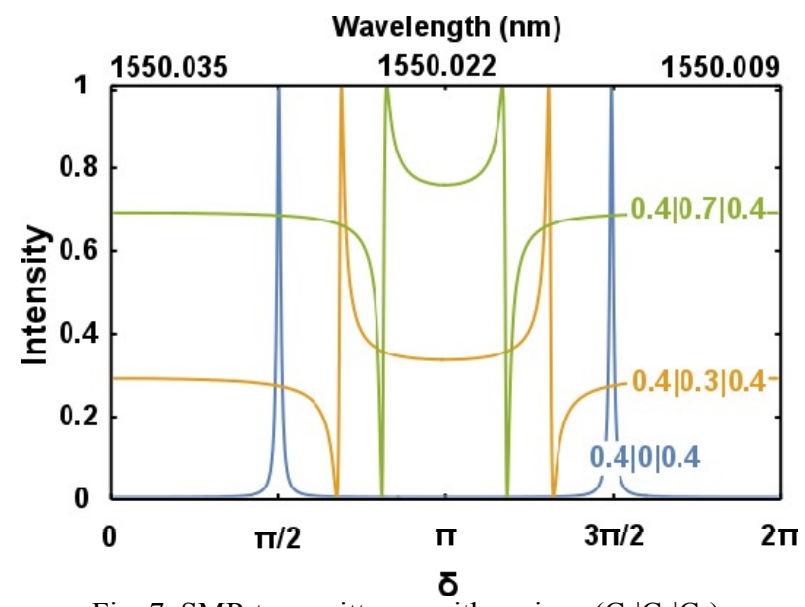

Fig. 7. SMR transmittance with various $\left(\mathrm{C}_{1}\left|\mathrm{C}_{2}\right| \mathrm{C}_{3}\right)$.

A symmetric meandering resonator (SMR) can be formed by cascading two MLMs in such a way that, the coupling can occur from both bottom and top of the MLM sections as shown in Fig. 6. Compared to the MR, the SMR shows a richer spectral response.

For geometric SMR path lengths of $20 \pi \mathrm{mm}, 30 \pi \mathrm{mm}$, and $40 \pi \mathrm{mm}$, the FSRs are $26 \mathrm{pm}, 17.4 \mathrm{pm}$, and $13 \mathrm{pm}$, respectively. For the following analysis, we have again chosen the minimum bending radius of $5 \mathrm{~mm}$ only, since it results in the minimum SMR optical path length leading to the maximum FSR $=26 \mathrm{pm}$. The SMR transmission intensities of the coupled field equations are plotted for different values of the coupling constants $\left(\mathrm{C}_{1}\left|\mathrm{C}_{2}\right| \mathrm{C}_{3}\right)$ in Fig. 7. For the case when $\left(\mathrm{C}_{1}\left|\mathrm{C}_{2}\right| \mathrm{C}_{3}\right)$ are set to $(0.4|0.4| 0)$, the transmission spectrum is similar to the MR with a straight section length $\theta_{L}=0$. For the $\mathrm{C}_{2} \neq 0$ case, $\mathrm{C}_{2}$ can provide control over the Rabi splitting between the Fano resonances [29]. 


\section{ANTISYMMETRIC MEANDERING RESONATOR}

The antisymmetric meandering resonator (AMR), can be realized by cascading a half MLM section after a full MLM in such a way that, two coupling points are created at the top and the bottom, as shown in Fig. 8. Coupled resonator induced transparency (CRIT) can be observed in the spectral response of the AMR, which can be used for signal buffering, biochemical sensing, and quantum signal processing [13].

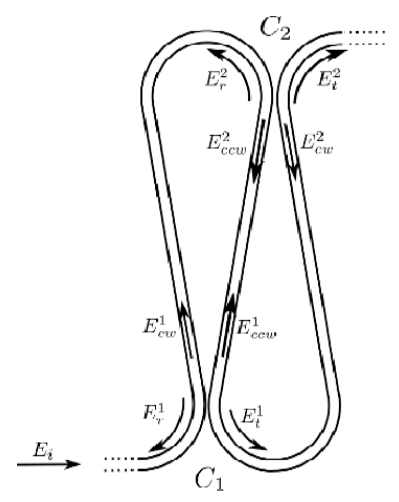

Fig. 8. AMR with $\mathrm{C}_{1}$ and $\mathrm{C}_{2}$.

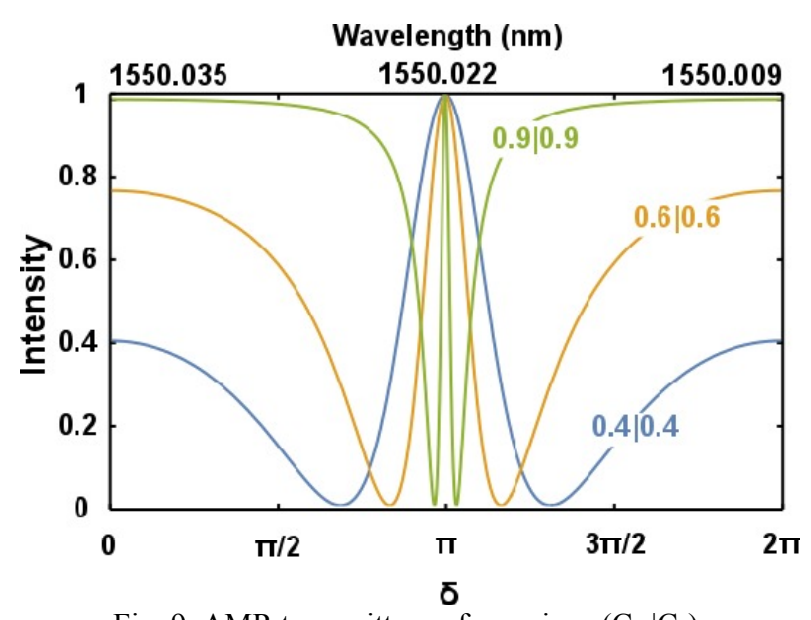

Fig. 9. AMR transmittance for various $\left(\mathrm{C}_{1} \mid \mathrm{C}_{2}\right)$.

Similar to the SMR, for the AMR we have chosen the minimum bending radius of $5 \mathrm{~mm}$ corresponding to the FSR of $26 \mathrm{pm}$. The transmission intensities of the AMR for various values of $\mathrm{C}_{1}=\mathrm{C}_{2}$ are plotted in Fig. 9. The coupled resonator induced transparency (CRIT) [30] effect is observed with a $\mathrm{Q}$ factor, that increases with the increasing value of the coupling constants $\mathrm{C}_{1}=\mathrm{C}_{2}$.

\section{SYMMETRIC MEANDERING DFB STRUCTURE}

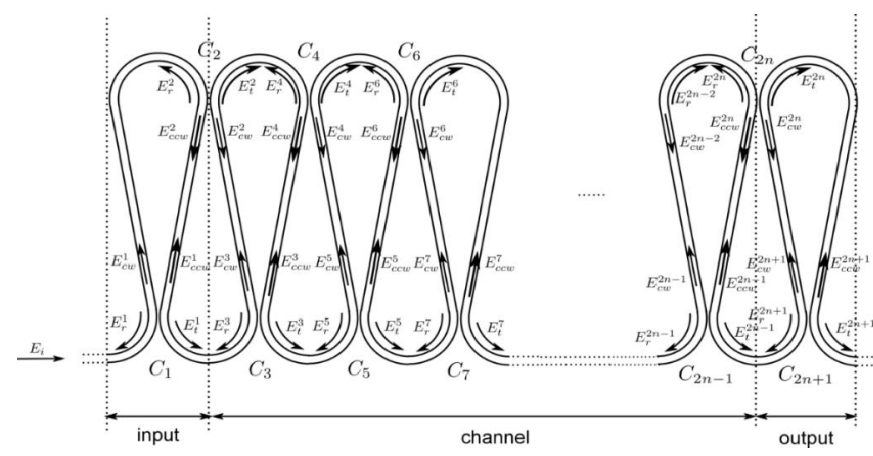

Fig. 10. SMDFB formed by MLM's with both top and bottom couplings.

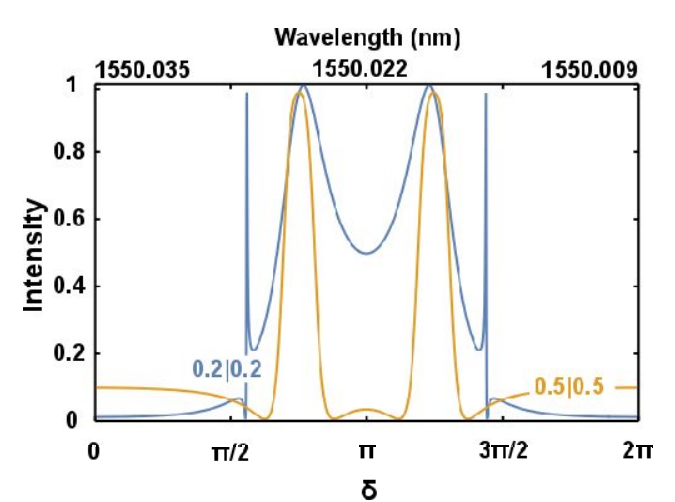

Fig. 11. SMDFB of 3 MLM transmittance for $\left(\mathrm{C}_{1,3,5} \mid \mathrm{C}_{2,4}\right)$.

The symmetric meandering distributed feedback structure (SMDFB) structure, shown in Fig. 10, was studied analytically by applying the transfer matrix method (TMM) to the inter- and intra-coupled field equations of the MLM's. SMDFB structures can be used as tunable optical delay lines [15]. For simplicity of calculations, the SMDFB is divided into channel MLMs (CMLMs) and, input / output boundary MLMs (BMLMs). While solving for the MDFB's transfer matrix equations simultaneously with the boundary equations, we end up with a couple of boundary conditions for the MDFB. 
There exist six CMLM transition expressions, and six internal field expressions on each side, connecting the BMLMs to the CMLMs. Two different boundary conditions are obtained while simultaneously solving the boundary equations for the MDFB. Corresponding to the four ports of a BMLM, there exist additional four field expressions for each BMLM, leading to a system of twenty-six coupled equations for the SMDFB.

Similar to SMR and AMR, we have chosen the minimum bending radius of $5 \mathrm{~mm}$ with a maximum FSR of $26 \mathrm{pm}$ for the SMDFB. In Fig. 11 for the cases, with equal coupling constants $\mathrm{C}=0.5$ and $\mathrm{C}=0.2$, the transmission spectrum of the 3-MLM SMDFB shows a mode splitting and a band-pass filter response. A similar behavior to Sagnac interferometer can also be achieved [31]. For the case when $\mathrm{C}=0.2$, Fano lineshapes are attained in the transmittance of the SMDFB.

\section{ANTISYMMETRIC MEANDERING DFB STRUCTURE}

Similar to SMDFB, BMLM's in the AMDFB structure is also comprised of four ports, so the CMLM equations and the input BMLM equations will remain the same [16] and the TMM analysis performed in the prior section is also valid here. AMDFB structure, shown in Fig. 12, was analyzed, while keeping $\mathrm{n}=2$, resulting in six ports (four at the top and two at the bottom). A similar method to the SMDFB was used to calculate the transmitted intensity. Again, similar to the SMDFB, we have chosen the minimum bending radius of $5 \mathrm{~mm}$ for a maximum FSR of $26 \mathrm{pm}$ for the AMDFB.

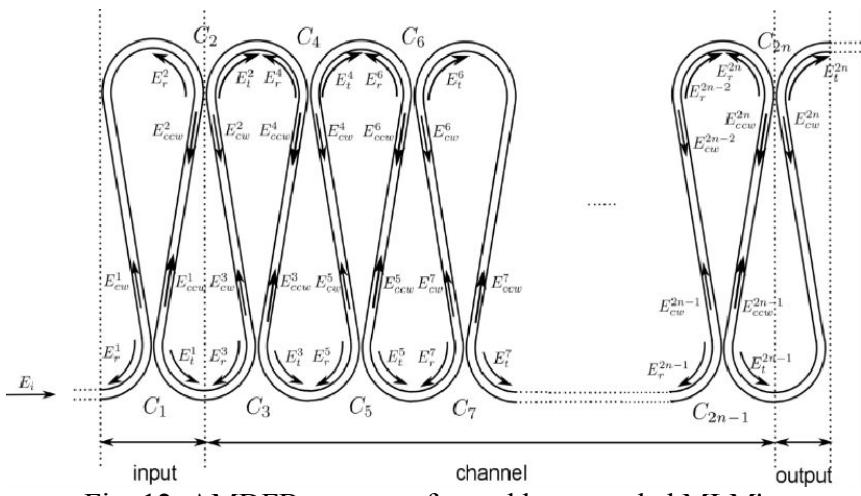

Fig. 12. AMDFB structure formed by cascaded MLM's.

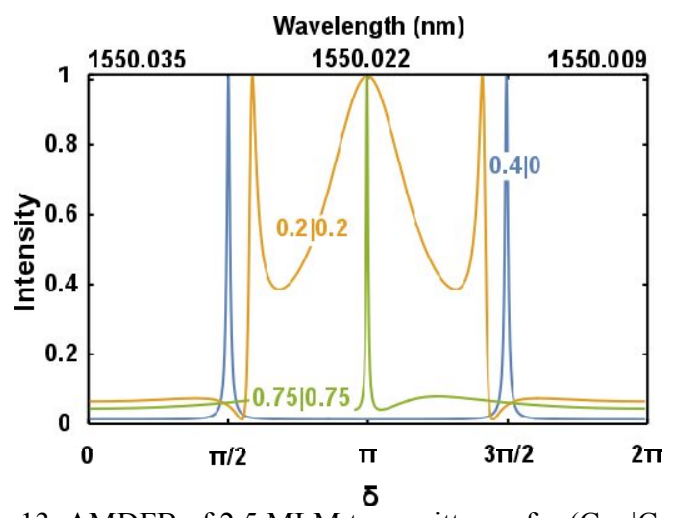

Fig. 13. AMDFB of 2.5 MLM transmittance for $\left(\mathrm{C}_{1,3} \mid \mathrm{C}_{2,4}\right)$.

In Fig. 13, for the cases, when $\left(\mathrm{C}_{1,3} \mid \mathrm{C}_{2,4}\right)=(0.4 \mid 0),(0.2 \mid 0.2)$ the transmittance spectrum of a 2.5 MLM AMDFB structure is similar to the SMDFB structure's response with its band-pass characteristics and mode splitting. For the case, when $\left(\mathrm{C}_{1,3} \mid \mathrm{C}_{2,4}\right)=(0.75 \mid 0.75)$, the transmission spectrum takes a winged Lorentzian lineshape.

\section{CONCLUSIONS}

Meandering lightwave SMF components are introduced, categorized, and numerically analyzed in the near-infrared telecommunication band at $1550 \mathrm{~nm}$. MLM can be considered as one of the building blocks for the photonic lightwave elements.

By using different combinations of the MLMs, we were able to analyze other fiber optic elements, such as MR, SMR, AMR, and their respective DFB structures. MR has Lorentzian peaks in the transmission spectra, and when more meanders are added, we see the Rabi splitting in the MDFB's 
transmission spectra. The transmission spectrum of SMR is richer compared to the MR, as we can observe both the Lorentzian and Fano lineshapes, while we tune the coupling coefficients. The SMDFB have a similar transmission spectrum with Lorentzian and Fano lineshapes. The AMR can be tuned to achieve the CRIT effect. The AMDFB shows Rabi splitting, and winged Lorentzian lineshapes.

With this variety of spectral responses, meandering waveguide SMF components can be used as filters and delay line elements in fiber optic communication, as well as sensor elements in fiber optic diagnostics.

\section{ACKNOWLEDGEMENTS}

Muhammad Zakwan would like to acknowledge the partial support of this work by the Higher Education Commission (HEC) of Pakistan.

\section{REFERENCES}

[1] Y. Liao, X. Wang, H. Yang, S. Wang and J. Wang, "Resonant Mode Characteristics of Microfiber Knot-Type Ring Resonator and Its Salinity Sensing Experiment," IEEE Photon. J., vol. 7, no. 4, pp. 1-8, Aug. 2015.

[2] A. Sulaiman, S. W. Harun and H. Ahmad, "Erbium-Doped Fiber Laser with a Microfiber Coupled to Silica Microsphere," IEEE Photon. J., vol. 4, no. 4, pp. 1065-1070, Aug. 2012.

[3] Z. Cheng, X. Chen, C. Y. Wong, K. Xu and H. K. Tsang, "Mid-infrared Suspended Membrane Waveguide and Ring Resonator on Silicon-on-Insulator," IEEE Photon. J., vol. 4, no. 5, pp. 1510-1519, Oct. 2012

[4] K. Xu, Y. Chen, C. Li, X. Chen, Z. Cheng, C. Y. Wong, H. K. Tsang, "An Ultracompact OSNR Monitor Based on an Integrated Silicon Microdisk Resonator," IEEE Photon. J., vol. 4, no. 5, pp. 1365-1371, Oct. 2012.

[5] J.-S. Hong and M. J. Lancaster, Microstrip Filters for RF/Microwave Applications, pp. 93-102, 2001, Wiley, Singapore.

[6] B. J. Rubin and B. Singh, "Study of meander line delay in circuit boards," IEEE Trans. Microw. Theory Techn., vol. 48, no. 9, pp. 1452-1460, Sep. 2000.

[7] D. Hou, Y.-Z. Xiong, W.-L. Goh, S. Hu, W. Hong, and M. Madihian, "130-GHz on-chip meander slot antennas with stacked dielectric resonators in standard CMOS technology," IEEE Trans. Antennas Propag., vol. 60, no. 9, pp. 4102-4109, Sep. 2012.

[8] S. K. Sheem and T. G. Giallorenzi, "Polarization effects on single-mode optical fiber sensors," Appl. Phys. Lett. vol. 35, no. 12, pp. 914-917, Dec. 1979.

[9] D. B. Mortimore, "Fiber loop reflectors," J. Lightwave Technol. vol. 6, no. 7, pp. 1217-1224, Jul. 1988.

[10] P. Urquhart, "Fiber lasers with loop reflectors," Appl. Opt. vol. 28, no. 17, pp. 3759-3767, Sep. 1989.

[11] P. Urquhart, "Compound optical-fiber-based resonators," J. Opt. Soc. Am. A, vol. 5, no. 6, pp. 803-812, Jun. 1988.

[12] H. Hodara, "My Forty-Year Adventure in the Wonderful World of Fiber Optics!," Fiber Integrated Opt., vol. 35, no. 5-6, pp. 252-266, Nov. 2016. 
[13] L.-J. Zhou, T. Ye, and J.-P. Chen, "Coherent interference induced transparency in self-coupled optical waveguide-based resonators," Opt. Lett. vol. 36, no. 1, pp. 13-15, Jan. 2011.

[14] Z. Zou, L.-J. Zhou, X.-M. Sun, J.-Y. Xie, H.-K. Zhu, L.-J. Lu, X.-W. Li, and J.-P. Chen, "Tunable two-stage self-coupled optical waveguide resonators," Opt. Lett. vol. 38, no. 8, pp. 1215-1217, Apr. 2013.

[15] J. Scheuer and O. Weiss, "The Serpentine Optical Waveguide: engineering the dispersion relations and the stopped light points," Opt. Express, vol. 19, no. 12, pp. 11517-11528, Jun. 2011.

[16] C. B. Dağ, M. A. Anıl, and A. Serpengüzel, "Meandering Waveguide Distributed Feedback Lightwave Circuits," J. Lightwave Technol. vol. 33, no. 9, pp. 1691-1702, May 2015.

[17] C. B. Dağ, M. A. Anıl, and A. Serpengüzel, "Novel distributed feedback lightwave circuit elements," in Proc. of the SPIE Symposia: "Smart Photonic and Optoelectronic Integrated Circuits XVII," Louay A. Eldada, El-Hang Lee, and Sailing He, Eds. published by SPIE, Bellingham, WA, USA, 9366, 93660A, Mar. 2015.

[18] C. B. Dağ, M. A. Anıl, and A. Serpengüzel, "Meandering Waveguide Distributed Feedback Lightwave Elements: Phasor Diagram Analysis," in Proc. of Progress In Electromagnetics Research Symposium, Prague, Czech Republic, published by The Electromagnetic Academy, Cambridge, MA, USA 1986-1990, Jul. 2015.

[19] H. Hodara and B. Crosignani, Eds. "Guided optical structures in the military environment: papers," Proc. of NATO-AGARD Electromagnetic Wave Propulsion Panel Specialists' Meeting, Istanbul, Turkey, published by AGARD, Neuilly-sur-Seine, France, pp. 1-310, 383, May 1986.

[20] N. J. Doran and D. Wood, "Nonlinear-optical loop mirror," Opt. Lett., vol. 13, no. 1, pp. 56-58, Jan. 1988.

[21] R. A. Bergh, H. C. Lefèvre, and H. J. Shaw, "All-single-mode fiber-optic gyroscope," Opt. Lett., vol. 6, no. 4 pp. 198-200, Apr. 1981.

[22] T. Wang, X.-H. Li, F.-F. Liu, W.-H. Long, Z.-Y. Zhang, L.-M. Tong, and Y.-K. Su, "Enhanced fast light in microfiber ring resonator with a Sagnac loop reflector," Opt. Express, vol. 18, no. 15, pp. 16156-16161, Jul. 2010.

[23] "Recommendation ITU-T G.652, Characteristics of a single-mode optical fibre and cable." International Telecommunication Union, 2010 Geneva, Switzerland.

[24] "Recommendation ITU-T G.657, Characteristics of a single-mode optical fibre and cable." International Telecommunication Union, 2012 Geneva, Switzerland.

[25] M. J. F. Digonnet and H. J. Shaw, "Analysis of a Tunable Single Mode Optical Fiber Coupler," IEEE Trans. Microw. Theory Techn. vol. 30, no. 4, pp. 592-600, Apr. 1982.

[26] D. Marcuse, "Reduction of dispersion in a multimode fiber waveguide with core index fluctuations," US 3,909,110, Sep. 1975.

[27] F. Koyama, "Recent Advances of VCSEL Photonics," J. Lightwave Technol., vol. 24, no. 12, pp. 4502-4513, Dec. 2006.

[28] W.-B. Yan, J.-F. Huang, and H. Fan, "Tunable single-photon frequency conversion in a Sagnac interferometer," Nature Sci. Rep., vol. 3, pp. 3555-1, Dec. 2013.

[29] X.-G. Tu, L. Y. Mario, and T. Mei, "Coupled Fano resonators," Opt. Express, vol. 18, no. 18, pp. 18820-18831, Aug. 2010.

[30] D. Smith, H. Chang, K. Fuller, A. Rosenberger, and R. Boyd, "Coupled-resonator-induced transparency," Phys. Rev. A., vol. 69, no. 6, pp. 063804-1, Jun. 2004.

[31] S. E. Miller, "Coupled Wave Theory and Waveguide Applications," Bell Syst. Tech. J., vol. 33, no. 3, pp. 661719, May 1954. 